\title{
A uniqueness result for the quasiconvex operator and first order PDEs for convex envelopes
}

\author{
E.N. Barron*, R.R. Jensen \\ Department of Mathematics and Statistics, Loyola University Chicago, Chicago, IL 60660, United States
}

Received 8 August 2012; accepted 21 February 2013

Available online 15 March 2013

\begin{abstract}
The operator involved in quasiconvex functions is $L(u)=\min _{|y|=1, y \cdot D u=0} y D^{2} u y^{T}$ and this also arises as the governing operator in a worst case tug-of-war (Kohn and Serfaty (2006) [7]) and principal curvature of a surface. In Barron et al. (2012) [4] a comparison principle for $L(u)=g>0$ was proved. A new and much simpler proof is presented in this paper based on Barles and Busca (2001) [3] and $L u$ and Wang (2008) [8]. Since $L(u) /|D u|$ is the minimal principal curvature of a surface, we show by example that $L(u)-g|D u|=0$ does not have a unique solution, even if $g>0$. Finally, we complete the identification of first order evolution problems giving the convex envelope of a given function.
\end{abstract}

(c) 2013 Elsevier Masson SAS. All rights reserved.

MSC: 35D40; 52A 41

Keywords: Quasiconvex; Principal curvature; Convex envelope

\section{Introduction}

In the paper [5] we introduced a degenerate elliptic, second order operator which is critical in studying functions with convex level sets, known as quasiconvex (or level-convex functions). The equations considered in [5] were of the form

$$
L(u) \equiv L\left(D u, D^{2} u\right)-g(x):=\min _{\{|y|=1, y \cdot D u=0\}} y \cdot D^{2} u y^{T}-g(x)=0, \quad x \in \Omega \subset \mathbb{R}^{n} .
$$

If $D u=0, L\left(0, D^{2} u\right)=\lambda_{1}\left(D^{2} u\right)$ is the first eigenvalue of $D^{2} u$. This equation is considered in the viscosity sense which can deal with problems like the lack of continuity of $L=L(p, M)$ in the vector $p \in \mathbb{R}^{n}$ at $p=0$. Viscosity solution theory employs upper and lower semicontinuous envelopes of $L$ to get around this problem. Another way to write $L$ is

$$
L(p, M)= \begin{cases}\lambda_{1}((I-p \otimes p) M), & p \neq 0 \\ \lambda_{1}(M), & p=0\end{cases}
$$

\footnotetext{
सर This project was supported by grant 1008602 from the National Science Foundation.

* Corresponding author.

E-mail addresses: ebarron@1uc.edu (E.N. Barron), rjensen@luc.edu (R.R. Jensen).
} 
If we replace the eigenvalue operator by the trace operator we do indeed get the mean curvature operator. In $\mathbb{R}^{2}, L$ is the mean curvature operator since the first eigenvalue will lead to the trace. We proved in [5] that $L(u)-g=0$ has a unique viscosity solution assuming $g>0$. If $g \equiv 0$, we proved that there is a unique quasiconvex viscosity solution of $L(u)=0$. That is the most one can say if $g=0$ because we constructed a simple example in [5] of nonuniqueness in general. Apart from the connection with quasiconvexity, we also established the connection with stochastic target problems, and some problems in differential geometry arising in mean curvature and tug-of-war games. In fact, in a worst case tug-of-war game introduced by Kohn and Serfaty [7], we start with the equation

$$
u(x)=\max _{b= \pm 1} \min _{|y|=1} u(x+\varepsilon b \cdot y)
$$

and expand to second order to get

$$
0=\max _{b= \pm 1} \min _{|y|=1} \varepsilon b y \cdot D u(x)+\frac{\varepsilon^{2}}{2} y \cdot D^{2} u \cdot y^{T}+o\left(\varepsilon^{2}\right) .
$$

Dividing by $\varepsilon^{2}>0$ implies

$$
0=\min _{|y|=1} \frac{1}{\varepsilon}|y \cdot D u(x)|+y \cdot D^{2} u(x) \cdot y^{T}+o_{\varepsilon}(1)
$$

and sending $\varepsilon \rightarrow 0$,

$$
0=\min _{\{|y|=1, y \cdot D u=0\}} y \cdot D^{2} u(x) \cdot y^{T} \equiv L(u) .
$$

$L$ is the limit operator in Kohn-Serfaty's tug-of-war which is a deterministic game.

In this paper we present a new and much shorter and simpler proof of the uniqueness theorem with $g>0$. It is based on an idea introduced in the important paper by Barles and Busca [3] and expanded upon in [8-10] in which one wants to exploit the fact that any nonconstant subsolution cannot attain a strict interior maximum. Lu and Wang showed that this approach could be used to treat the infinity Laplacian with inhomogeneous signed terms as well as more general nonlinear degenerate elliptic operators.

One question which arose in [5] is whether or not one has uniqueness of the principal curvature equation. This equation involves our quasiconvexity operator and is given by $L(u) /|D u|$. The question is whether there is a unique viscosity solution of $L(u)-g(x)|D u|=0$. The answer is negative even with $g>0$ as we show in a simple example in the next section.

We also complete the introduction of equations generating the convex envelope and quasiconvex envelopes of a given function $g$. The second order obstacle problem giving the quasiconvex envelope was studied in [5] and is given by $\min \{L(u), g-u\}=0$. This was motivated by a paper by Oberman [12] and Oberman and Silvestre [11] in which they showed that the solution of $\max \left\{-\lambda_{1}\left(D^{2} u\right), u-g\right\}=0$ is $g^{* *}$, the convex envelope of $g$. In the present paper, we formulate the first order obstacle problems giving the convex and quasiconvex envelopes. This is based on the fact that first order conditions are sufficient to determine if a function is convex, or quasiconvex, and is an extension of a paper involving first order conditions in [4]. The new wrinkle is that the first order obstacle problems for the envelopes are not local. Unfortunately, the most we can say for the direct first obstacle problem is that the convex envelope is the maximal subsolution since the obstacle problem itself does not have unique solutions.

Thus we are led to consider an iterative scheme to construct the convex envelope motivated by a similar construction for quasiconvex envelopes in [4]. The scheme considers the nonlocal equation

$$
\begin{aligned}
& u_{n+1}(x)+\max _{z \in \bar{\Omega}}\left(D u_{n+1}(x) \cdot(z-x)-u_{n+1}(z)\right)=u_{n}(x), \\
& u_{0}(x)=g(x) .
\end{aligned}
$$

We prove that $\lim _{n \rightarrow \infty} u_{n}(x)=g^{* *}(x)$.

\section{The quasiconvexity operator}

Let $\Omega$ be an open bounded domain in $\mathbb{R}^{n}, B_{1}(0) \subset \mathbb{R}^{n}$ the surface of the unit ball, and $\mathcal{S}(n)$ the space of $n \times n$ symmetric matrices. Throughout this paper we assume $g: \bar{\Omega} \rightarrow \mathbb{R}$ is a continuous function. Consider the equation

$$
L(u)-g(x)=0, \quad x \in \Omega, \quad u(x)=h(x) \in C(\partial \Omega), \quad x \in \partial \Omega,
$$


where $L(u)=L\left(D u, D^{2} u\right)$ and

$$
L(p, M):= \begin{cases}\min \left\{y M y^{T} \mid y \in B_{1}(0), y \cdot p=0\right\}, & p \neq 0, \\ \min \left\{y M y^{T} \mid y \in B_{1}(0)\right\}, & p=0 .\end{cases}
$$

By definition $L(0, M)$ is the first eigenvalue, $\lambda_{1}(M)$ of the symmetric matrix $M \in \mathcal{S}(n)$.

We also have the following formula.

Lemma 2.1. We have for any $(p, M) \in B_{1}(0) \times \mathcal{S}(n)$,

$$
L(p, M)=\lambda_{1}((I-p \otimes p) M)
$$

the first eigenvalue of the matrix $(I-p \otimes p) M$.

From the lemma we see that

$$
L\left(D u, D^{2} u\right)= \begin{cases}\lambda_{1}\left(\left(I-\frac{D u \otimes D u}{|D u|^{2}}\right) D^{2} u\right), & \text { if } D u \neq 0 \\ \lambda_{1}\left(D^{2} u\right), & \text { if } D u=0 .\end{cases}
$$

For viscosity solutions we need the upper and lower semicontinuous envelopes of $L$ given in the following lemma.

Lemma 2.2. Let $p \in \mathbb{R}^{n}, M \in S(n)$, and $L(p, M)$ given in (2.1). Then $L(p, M)$ is lower semicontinuous,

$$
L_{*}(p, M) \equiv \liminf _{q \rightarrow p, N \rightarrow M} L(q, N)=L(p, M), \quad p \in \mathbb{R}^{n}, M \in S(n),
$$

and the upper semicontinuous envelope of $L$, labeled $L^{*}$ is

$$
\begin{aligned}
L^{*}(p, M) & = \begin{cases}\min \left\{y M y^{T} \mid y \in B_{1}(0), y \cdot p=0\right\}, & \text { if } p \neq 0 ; \\
\sup _{|q|=1} \min \left\{y M y^{T} \mid y \in B_{1}(0), y \cdot q=0\right\}, & \text { if } p=0,\end{cases} \\
& = \begin{cases}\lambda_{1}\left(\left(I-\frac{p \otimes p}{|p|^{2}}\right) M\right), & \text { if } p \neq 0 ; \\
\sup _{|q|=1} \lambda_{1}((I-q \otimes q) M), & \text { if } p=0 .\end{cases}
\end{aligned}
$$

With this lemma we have the definition.

Definition 2.3. A locally bounded function $u: \Omega \rightarrow \mathbb{R}$ is a viscosity solution of $L\left(D u, D^{2} u\right)-g(x)=0$ if 1. $u$ is a subsolution, i.e., if $x_{0} \in \arg \max u^{*}-\varphi$ implies

$$
g\left(x_{0}\right) \leqslant L^{*}\left(D \varphi\left(x_{0}\right), D^{2} \varphi\left(x_{0}\right)\right)= \begin{cases}\min \left\{y D^{2} \varphi\left(x_{0}\right) y^{T} \mid y \in B_{1}(0), y \cdot D \varphi\left(x_{0}\right)=0\right\}, & \text { if } D \varphi\left(x_{0}\right) \neq 0 ; \\ \sup _{|q|=1} \min \left\{y D^{2} \varphi\left(x_{0}\right) y^{T} \mid y \in B_{1}(0), y \cdot q=0\right\}, & \text { if } D \varphi\left(x_{0}\right)=0 .\end{cases}
$$

2. $u$ is a supersolution, i.e., if $x_{0} \in \arg \min u_{*}-\varphi$ implies

$$
g\left(x_{0}\right) \geqslant L\left(D \varphi\left(x_{0}\right), D^{2} \varphi\left(x_{0}\right)\right)= \begin{cases}\min \left\{y D^{2} \varphi\left(x_{0}\right) y^{T} \mid y \in B_{1}(0), y \cdot D \varphi\left(x_{0}\right)=0\right\}, & \text { if } D \varphi\left(x_{0}\right) \neq 0 ; \\ \lambda_{1}\left(D^{2} \varphi\left(x_{0}\right)\right), & \text { if } D \varphi\left(x_{0}\right)=0,\end{cases}
$$

where $u^{*}$ and $u_{*}$ denote the upper and lower semicontinuous envelopes of $u$, respectively. The boundary condition $u=h$ on $\partial \Omega$, means $x \in \arg \max _{\partial \Omega} u-\varphi$ implies $\max \left\{L^{*}(\varphi)-g, h-u^{*}\right\} \geqslant 0$ and $x \in \arg \min _{\partial \Omega} u-\varphi$ implies $\min \left\{L_{*}(\varphi)-g, h-u_{*}\right\} \leqslant 0$.

Theorem 2.4. Assume that $u$ is an upper semicontinuous subsolution bounded from above of $L(u)-g(x) \geqslant 0$ and $v$ is a lower semicontinuous supersolution bounded from below of $L(v)-g(x) \leqslant 0$. Assume $g(x)>0$ and $g \in C(\bar{\Omega})$. Assume $u(x) \leqslant v(x)$ on $\partial \Omega$. Then $u(x) \leqslant v(x)$ on $\bar{\Omega}$.

Proof. Begin by observing that for any $\sigma>0$ we may set $u_{\sigma}=u-\sigma\left(\max _{x \in \partial \Omega} u(x)-u(x)\right)$ and then $u_{\sigma} \leqslant u \leqslant v$ on $\partial \Omega$ as well as

$$
L\left(u_{\sigma}\right)=(1+\sigma) L(u) \geqslant(1+\sigma) g(x):=g_{\sigma}(x)>g(x) .
$$


Step 1 . We want to transform $u_{\sigma}$ and $v$ into semiconvex functions. The way to do that is to take the convolution of each function:

$$
v_{\varepsilon}(x)=\inf _{z \in \bar{\Omega}}\left\{v(z)+\frac{1}{2 \varepsilon}|x-z|^{2}\right\} \quad \text { and } \quad u_{\sigma}^{\varepsilon}(x)=\sup _{y \in \bar{\Omega}}\left\{u_{\sigma}(y)-\frac{1}{2 \varepsilon}|x-y|^{2}\right\} .
$$

Let $K=\sup _{x \in \Omega} u(x) \vee v(x)$, and set $\Omega_{\delta}=\{x \in \Omega \mid \operatorname{dist}(x, \partial \Omega)>\delta\}$. It is standard to prove the following lemma (see [8] for details for a general inhomogeneous degenerate elliptic operator).

Lemma 2.5. If $u_{\sigma}$ is a subsolution of $L\left(u_{\sigma}\right)-g_{\sigma} \geqslant 0$ and $v$ is a supersolution of $L(v)-g \leqslant 0$, then $u_{\sigma}^{\varepsilon}$ is a subsolution of

$$
L\left(u_{\sigma}^{\varepsilon}\right) \geqslant g_{\sigma, \varepsilon}(x):=(1+\sigma) \inf \{g(x+z)|| z \mid \leqslant 2 \sqrt{K \varepsilon}\},
$$

and $v_{\varepsilon}$ is a supersolution of

$$
L\left(v_{\varepsilon}\right) \leqslant g^{\varepsilon}(x):=\sup \{g(x+z)|| z \mid \leqslant 2 \sqrt{K \varepsilon}\}
$$

for $x \in \Omega_{\delta}, \delta=3 \sqrt{K \varepsilon}$. Furthermore, $u_{\sigma}^{\varepsilon}$ is semiconvex and $v_{\varepsilon}$ is semiconcave, and they converge as $\varepsilon \rightarrow 0+$ to $u_{\sigma}, v$, respectively, and both $u_{\sigma}^{\varepsilon}$ and $v_{\varepsilon}$ are differentiable at max points of $u_{\sigma}^{\varepsilon}-v_{\varepsilon}$. In addition $g_{\sigma, \varepsilon}$ and $g^{\varepsilon}$ are continuous functions on $\Omega_{\delta}$.

We have shown that $u_{\sigma}^{\varepsilon}$ and $-v_{\varepsilon}$ are semiconvex, and $u_{\sigma}^{\varepsilon}$ is a subsolution of

$$
L\left(u_{\sigma}^{\varepsilon}\right)-g_{\sigma, \varepsilon} \geqslant 0, \quad x \in \Omega_{\delta},
$$

and $v^{\varepsilon}$ is a supersolution of

$$
L\left(v^{\varepsilon}\right)-g^{\varepsilon} \leqslant 0, \quad x \in \Omega_{\delta} .
$$

Since $g>0$ we may also assume

$$
g_{\sigma, \varepsilon}(x)=(1+\sigma) g_{\varepsilon}(x)>g^{\varepsilon}(x), \quad x \in \Omega_{\delta}
$$

for all $\varepsilon>0$ and $\sigma>0$ sufficiently small depending only on $\|g\|_{L^{\infty}\left(\bar{\Omega}_{\delta}\right)}$. Now we drop the $\varepsilon$ in our notation and assume that $u_{\sigma}$ is a semiconvex subsolution of $L\left(u_{\sigma}\right)-g_{\sigma} \geqslant 0, v$ is a supersolution of $L(v) \leqslant g$ in $\Omega$ and $g_{\sigma}>g>0$.

Step 2. Suppose $u_{\sigma}\left(x_{0}\right)>v\left(x_{0}\right)$ and $x_{0} \in \arg \max _{\bar{\Omega}}\left(u_{\sigma}-v\right)$.

There is a $\delta>0$ so that $v\left(x_{0}\right)<u_{\sigma}\left(x_{0}+\xi\right)$ for any $|\xi|<\delta$ and $u_{\sigma}(x+\xi)<v(x), x \in \Omega \backslash \Omega_{\delta}$ as well as $g_{\sigma}(x+\xi)>$ $g(x), x \in \Omega_{\delta}$. Make the definitions for $\varepsilon>0, \xi \in B_{\delta}(0)$,

$$
\begin{aligned}
& w(x, y):=u_{\sigma}(x+\xi)-v(y)-\frac{1}{2 \varepsilon}|x-y|^{2}, \quad \varepsilon>0,|\xi|<\delta, \\
& M(0):=\max _{x \in \bar{\Omega}}\left(u_{\sigma}-v\right)=u_{\sigma}\left(x_{0}\right)-v\left(x_{0}\right)>0, \\
& M(\xi):=\max _{x \in \Omega_{\delta}}\left(u_{\sigma}(x+\xi)-v(x)\right)>0, \\
& M(\varepsilon, \xi):=\max _{(x, y) \in \bar{\Omega}_{\delta} \times \bar{\Omega}_{\delta}} w(x, y), \\
& \left(x_{\varepsilon, \xi}, y_{\varepsilon, \xi}\right) \in \underset{(x, y) \in \bar{\Omega}_{\delta} \times \bar{\Omega}_{\delta}}{\arg \max } w(x, y), \\
& M(\varepsilon, \xi)=u_{\sigma}\left(x_{\varepsilon, \xi}+\xi\right)-v\left(y_{\varepsilon, \xi}\right)-\frac{1}{2 \varepsilon}\left|x_{\varepsilon, \xi}-y_{\varepsilon, \xi}\right|^{2} .
\end{aligned}
$$

Refer to Crandall, Ishii and Lions [6] for the proofs of the following results.

$$
M(\varepsilon, \xi) \rightarrow M(\xi), \quad \frac{1}{2 \varepsilon}\left|x_{\varepsilon, \xi}-y_{\varepsilon, \xi}\right|^{2} \rightarrow 0, \quad u_{\sigma}\left(x_{\varepsilon, \xi}+\xi\right)-v\left(y_{\varepsilon, \xi}\right) \rightarrow M(\xi), \quad \text { as } \varepsilon \rightarrow 0+.
$$

Also,

$$
M(\xi)>0 \geqslant \max _{\partial \Omega_{\delta}}\left(u_{\sigma}(x+\xi)-v(x)\right) \Longrightarrow \quad x_{\varepsilon, \xi}, y_{\varepsilon, \xi} \in \Omega_{1} \Subset \Omega_{\delta}, \quad \forall \text { small } \varepsilon>0 .
$$


There are symmetric matrices $X, Y$ (which depend on $\varepsilon, \xi$ ) so that

$$
\left(\frac{x_{\varepsilon, \xi}-y_{\varepsilon}, \xi}{\varepsilon}, X\right) \in D^{2+} u\left(x_{\varepsilon, \xi}+\xi\right), \quad\left(\frac{x_{\varepsilon, \xi}-y_{\varepsilon, \xi}}{\varepsilon}, Y\right) \in D^{2-} v\left(y_{\varepsilon, \xi}\right)
$$

and

$$
-\frac{3}{\varepsilon}\left[\begin{array}{cc}
I & 0 \\
0 & I
\end{array}\right] \leqslant\left[\begin{array}{cc}
X & 0 \\
0 & -Y
\end{array}\right] \leqslant \frac{3}{\varepsilon}\left[\begin{array}{cc}
I & -I \\
-I & I
\end{array}\right]
$$

in the sense of matrices. This implies $X \leqslant Y$.

Now we consider cases corresponding to zero gradient, or nonzero gradient.

Case 1 (Nonzero gradient): There are $\xi \in B_{\delta}(0)$ and a subsequence (still denoted by $\varepsilon$ ) $\varepsilon \rightarrow 0$ so that $x_{\varepsilon, \xi}-$ $\boldsymbol{y}_{\varepsilon, \xi} \neq \mathbf{0}, \forall \varepsilon>\mathbf{0}$.

In this case, since $u_{\sigma}$ is a subsolution and $v$ is a supersolution and the gradient is nonzero,

$$
\begin{aligned}
g_{\sigma}\left(x_{\varepsilon, \xi}+\xi\right) & \leqslant L^{*}\left(u\left(x_{\varepsilon, \xi}+\xi\right)\right)=\min \left\{q \cdot X \cdot q^{T}|| q \mid=1, q \cdot \frac{x_{\varepsilon, \xi}-y_{\varepsilon, \xi}}{\varepsilon}=0\right\} \\
& \leqslant \min \left\{q \cdot Y \cdot q^{T}|| q \mid=1, q \cdot \frac{x_{\varepsilon, \xi}-y_{\varepsilon, \xi}}{\varepsilon}=0\right\} \\
& =L^{*}\left(v\left(y_{\varepsilon, \xi}\right)\right) \leqslant g\left(y_{\varepsilon, \xi}\right)
\end{aligned}
$$

and this gives us

$$
g_{\sigma}\left(x_{\varepsilon, \xi}+\xi\right) \leqslant g\left(y_{\varepsilon, \xi}\right) .
$$

Since $\left|x_{\varepsilon, \xi}-y_{\varepsilon, \xi}\right| \rightarrow 0, \varepsilon \rightarrow 0+$ we know that on a subsequence of $\{\varepsilon\}$ we have $x_{\varepsilon, \xi} \rightarrow x_{\xi}$ and $y_{\varepsilon, \xi} \rightarrow x_{\xi}$. Hence, sending $\varepsilon \rightarrow 0+$ we get $g_{\sigma}\left(x_{\xi}+\xi\right)=(1+\sigma) g\left(x_{\xi}+\xi\right) \leqslant g\left(x_{\xi}\right)$ which is a contradiction to $g_{\sigma}(x+\xi)>g(x), x \in \Omega_{\delta}$.

Case 2 (Zero gradient): For every $\xi \in B_{\delta}(0), x_{\varepsilon, \xi}=y_{\varepsilon, \xi}$ for all small $\varepsilon>0$ and a pair $\left(x_{\varepsilon, \xi}, y_{\varepsilon, \xi}\right)$.

Observe, that in these circumstances

$$
\begin{aligned}
(1+\sigma) g\left(x_{\varepsilon, \xi}+\xi\right) & \leqslant L^{*}\left(u_{\sigma}\left(x_{\varepsilon, \xi}+\xi\right)\right) \\
& =\max _{|q|=1} \min _{|y|=1, q \cdot y=0} y X y^{T} \\
& \leqslant \max _{|q|=1|y|=1, q \cdot y=0} \min _{\mid y y^{T}} \\
& =L^{*}\left(v\left(y_{\varepsilon, \xi}\right)\right)
\end{aligned}
$$

and that is as far as we can go with this since we know $L_{*}\left(v\left(y_{\varepsilon, \xi}\right)\right) \leqslant g\left(y_{\varepsilon, \xi}\right)$ but not $L^{*}$. We need another approach.

In case 2 , we have

$$
M(\varepsilon, \xi)=u_{\sigma}\left(x_{\varepsilon, \xi}+\xi\right)-v\left(x_{\xi}\right)=M(\xi) .
$$

Recall that $z \mapsto u_{\sigma}(z+\xi)$ and $z \mapsto-v(z)$ are both semiconvex and $x_{\varepsilon, \xi}$ is the maximum point of $u_{\sigma}(z+\xi)-v(z)$. Therefore each function is differentiable at $x_{\varepsilon, \xi}$. Since $x_{\varepsilon, \xi} \in \arg \max w$ we have for any $y \in \bar{\Omega}_{\delta}$

$$
\begin{gathered}
u_{\sigma}\left(x_{\varepsilon, \xi}+\xi\right)-v\left(x_{\varepsilon, \xi}\right) \geqslant u_{\sigma}(y+\xi)-v\left(x_{\varepsilon, \xi}\right)-\frac{1}{2 \varepsilon}\left|x_{\varepsilon, \xi}-y\right|^{2} \\
\Longrightarrow u_{\sigma}\left(x_{\varepsilon, \xi}+\xi\right) \geqslant u_{\sigma}(y+\xi)-\frac{1}{2 \varepsilon}\left|x_{\varepsilon, \xi}-y\right|^{2}
\end{gathered}
$$

for all small $\varepsilon>0$. That implies that $D u_{\sigma}\left(x_{\varepsilon, \xi}+\xi\right)=D v\left(x_{\varepsilon, \xi}\right)=0$.

Next we claim: $M(\xi)=M(0)$ for all $\xi$ sufficiently small. 
To prove the claim choose $\xi, \eta \in B_{\delta}(0)$. By definition of the point $x_{\varepsilon, \xi}$

$$
\begin{aligned}
M(\xi) & =u_{\sigma}\left(x_{\varepsilon, \xi}+\xi\right)-v\left(x_{\varepsilon, \xi}\right) \\
& \geqslant u_{\sigma}\left(x_{\varepsilon, \eta}+\xi\right)-v\left(x_{\varepsilon, \eta}\right) \\
& =M(\eta)+u_{\sigma}\left(x_{\varepsilon, \eta}+\xi\right)-u_{\sigma}\left(x_{\varepsilon, \eta}+\eta\right) \\
& \geqslant M(\eta)-o(|\xi-\eta|)
\end{aligned}
$$

since $D u_{\sigma}\left(x_{\varepsilon, \eta}+\eta\right)=0$. We may reverse the roles of $\xi, \eta$ and use the fact that $M(\xi)$ is Lipschitz (and so differentiable almost everywhere) to conclude that $D M(\xi)=0$ almost everywhere in $B_{\delta}(0)$. But then $M(\xi)$ is constant in $B_{\delta}(0)$ and $M(\xi)=M(0)$ for all $x \in B_{\delta}(0)$, and $\delta>0$ small.

Consider the point $x_{0}$. At this point we have that $g_{\sigma}\left(x_{0}\right)=(1+\sigma) g\left(x_{0}\right)>0$. Then $L\left(u_{\sigma}\right) \geqslant(1+\sigma) g(x)>$ $g(x)>0$ in a neighborhood of $x_{0}$. If $|\xi|<\delta$, we have from the fact $M(\xi)=M(0)$

$$
u_{\sigma}\left(x_{0}+\xi\right)-v\left(x_{0}\right) \leqslant u_{\sigma}\left(x_{\varepsilon, \xi}+\xi\right)-v\left(x_{\varepsilon, \xi}\right)=u_{\sigma}\left(x_{0}\right)-v\left(x_{0}\right) \quad \Longrightarrow \quad u_{\sigma}\left(x_{0}+\xi\right) \leqslant u_{\sigma}\left(x_{0}\right) .
$$

This says $x_{0} \in \Omega_{\delta}$ is a local maximum point of $u_{\sigma}$. That conclusion will contradict the following lemma when applied to the domain $\Omega_{\delta}$.

Lemma 2.6. Let $u \in C(\bar{\Omega})$ be a semiconvex subsolution of $L(u)-g \geqslant 0$, with $g \in C(\bar{\Omega}), g>0$. Then

$$
\max _{x \in \bar{\Omega}} u(x)=\max _{x \in \partial \Omega} u(x)>u(x), \quad \text { for any } x \in \Omega .
$$

That is, u cannot attain an interior local maximum.

Proof. Assume there is a point $x_{0} \in \Omega$ with $x_{0} \in \arg \max _{x \in \Omega} u(x)$ and that $u\left(x_{0}\right)>\max _{\partial \Omega} u$. If that is the case, then there is a smooth function $\varphi(x) \equiv u\left(x_{0}\right)$ such that $u-\varphi$ has a zero (local) maximum at $x_{0}$. Since $D \varphi\left(x_{0}\right)=$ $D^{2} \varphi\left(x_{0}\right)=0$, we have

$$
L^{*}\left(\varphi\left(x_{0}\right)\right)-g\left(x_{0}\right)=\sup _{|p|=1} \min \left\{y D^{2} \varphi\left(x_{0}\right) y^{T}|| y \mid=1, p \cdot y=0\right\}=-g\left(x_{0}\right)<0,
$$

a contradiction.

Finally, we have shown that $u_{\sigma}(x) \leqslant v(x), x \in \Omega_{\delta}$ for all small $\sigma>0$ and $\delta$. Consequently, sending $\sigma \rightarrow 0$ and $\delta \rightarrow 0$ we conclude that $u \leqslant v$ in $\bar{\Omega}$.

Example 2.7. The problem $\frac{L(u)}{|D u|}=g(x)$ is the problem of prescribed principal curvature. The question arises as to whether this problem possesses a unique solution. The answer is no, in general. This example shows that in general the problem $L(u)-g(x)|D u|=0$ with given boundary values does not have a unique solution.

Define $\Omega=\{|x|<2\} \subset \mathbb{R}^{n}$, and

$$
g(x)= \begin{cases}1, & \text { if } 0 \leqslant|x| \leqslant 1 \\ \frac{1}{|x|}, & \text { if } 1 \leqslant|x| \leqslant 2 .\end{cases}
$$

The function $g>0$ is continuous. Now take $\varphi:[0,2] \rightarrow \mathbb{R}$

$$
\varphi(r)= \begin{cases}0, & \text { if } r \leqslant 1 ; \\ (r-1)^{3}, & \text { if } 1 \leqslant r \leqslant 2\end{cases}
$$

and $u(x):=\varphi(|x|), x \in \bar{\Omega}$. The boundary data is $u(x)=1,|x|=2$. It is a calculus exercise to show that $L(u, D u)-$ $g(x)|D u|=0$ in $\Omega$. However, it is trivial that $v(x) \equiv 1$ is also a solution. In fact $u$ and $v$ as classical solutions are also viscosity solutions.

It is actually true, as the reader can verify, that if we take $\varphi(r)$ to be any smooth strictly increasing function on $[1,2]$, with $\varphi(2)=1$, the function $u(x)=\varphi(|x|)$ will be a solution.

This example shows that the equation of prescribed principal curvature $\frac{L(u)}{|D u|}=g$, is not enough to completely specify the function $u$ even if we assume $g(x)>0$. On the other hand it is feasible that some conditions on $g$ could 
lead to uniqueness (such as $g$ as a positive constant). However, it should be noted that our proof of uniqueness fails for the problem $L(u)-c|D u|=0, c>0$. We leave this as an open problem. Furthermore, determining conditions yielding uniqueness for equations of the form $L(u)-g(x, D u)=0$ is also open.

\section{The first order equation for the convex envelope}

In this section we will take $\Omega \subset \mathbb{R}^{n}$ to be a convex and bounded domain and $g: \bar{\Omega} \rightarrow \mathbb{R}$ a continuous function. The greatest convex minorant of $g$ is the function

$$
g^{* *}(x)=\sup _{p \in \bar{\Omega}}\left\{p \cdot x-g^{*}(p)\right\}, \quad \text { where } g^{*}(p)=\sup _{x \in \bar{\Omega}}\{p \cdot x-g(x)\} .
$$

It is also known that

$$
g^{* *}(x)=\sup \left\{\lambda(x) \mid \lambda(x)=\alpha \cdot x+\beta, \exists \alpha \in \mathbb{R}^{n}, \beta \in \mathbb{R}, \lambda(x) \leqslant g(x), \forall x \in \bar{\Omega}\right\},
$$

i.e., $g^{* *}(x)$ is the pointwise supremum of all linear functions lying below $g(x)$. It was proved in [11] and [12] that $g^{* *}$ is the viscosity solution of the second order obstacle problem

$$
\min \left\{\lambda_{1}\left(D^{2} u\right), g-u\right\}=0, \quad x \in \Omega, u=g, x \in \partial \Omega .
$$

Our goal is to find a first order partial differential equation construction of $g^{* *}$ which arises in a completely natural way from the first order convexity condition. We begin by showing the following.

Lemma 3.1. (1) Let $u: \bar{\Omega} \rightarrow \mathbb{R}$ be upper semicontinuous. Then $u$ is convex if and only if for any $\varphi \in C^{2}$ and $x \in$ $\arg \max (u-\varphi)$ we have

$$
u(x)+\max _{z \in \bar{\Omega}} D \varphi(x) \cdot(z-x)-u(z)=0 .
$$

(2) Let $u: \bar{\Omega} \rightarrow \mathbb{R}$ be lower semicontinuous. Then $u$ is convex if and only if for any $\varphi \in C^{2}$ and $x_{0} \in \arg \min (u-\varphi)$ we have

$$
u(x)+\max _{z \in \bar{\Omega}} D \varphi(x) \cdot\left(z-x_{0}\right)-u(z)=0 .
$$

Proof. Suppose $u$ is convex and $x \in \arg \max (u-\varphi)$. If a smooth function contacts $u$ from above at $x$ it must be the case that $D u(x)$ exists and $D \varphi(x)=D u(x)$.

Indeed, let $q \in \partial u(x)$, the convex subdifferential of $u$, be arbitrary. Then

$$
u(x)+q \cdot(y-x) \leqslant u(y) \leqslant \varphi(y)=u(x)+D \varphi(x) \cdot(y-x)+o(|y-x|) .
$$

Sending $|y-x| \rightarrow 0$ gives us that $q=D \varphi(x)$ and thus $u$ is differentiable at $x$ with $D u(x)=D \varphi(x)$. Then, convexity says

$$
u(x)+D u(x) \cdot(z-x) \leqslant u(z) \quad \forall z \in \bar{\Omega} .
$$

This gives (3.2). Note that we do obtain equality in (3.2) by taking $z=x$.

Conversely, suppose (3.2) holds in viscosity sense but $u$ is not convex. We may assume

$$
x=\left(x_{1}, 0, \ldots, 0\right), \quad z=\left(z_{1}, 0, \ldots, 0\right), \quad x_{1}<z_{1},
$$

and that the set of maximizers of $u$ along $[x, z]$ is a closed set $Z$ in the relative interior of $[x, z]$. Let the maximum value be $M$. By upper semicontinuity of $u$, there exists a compact neighborhood $V$ of $0 \in \mathbb{R}^{n-1}$ such that $u\left(x_{1}, V\right)<M$, $u\left(z_{1}, V\right)<M$. Let

$$
\varphi_{i}\left(x_{1}, x_{2}, \ldots, x_{n}\right)=\frac{1}{i} x_{1}+i\left(x_{2}^{2}+\cdots+x_{n}^{2}\right) .
$$

The epigraphical limit of $u-\varphi$ is given by $u$ if $x_{2}=\cdots=x_{n}=0,-\infty$ otherwise. The maximum of the limit, relative to the compact set $[x, z]+(0, V)$, is attained on $Z$. Then, for some large enough $i$, the maximum of $u-\varphi_{i}$ 
relative to $[x, z]+(0, V)$ is attained at some $\xi=\left(\xi_{1}, \ldots, \xi_{n}\right)$ with $\xi_{1} \in\left(x_{1}, z_{1}\right)$, and $u(\xi)>u\left(z_{1}, V\right)$. Considering $y=\left(z_{1}, \xi_{2}, \ldots, \xi_{n}\right)$ yields

$$
u(\xi)-u(y)+D \varphi_{i}(\xi) \cdot(y-\xi)=u(\xi)-u(y)+\frac{1}{i}\left(z_{1}-\xi_{1}\right)>0 .
$$

This contradicts the fact that $u$ is a subsolution of (3.2).

The proof of (2) follows from the fact that a lower semicontinuous function is convex if and only if

$$
u(z) \geqslant u(x)+p \cdot(z-x), \quad \forall x, y \in \bar{\Omega}
$$

for every $p \in \partial u(x)$. For any $p \in \partial u(x)$, there is $\varphi \in C^{1}$ such that $u-\varphi$ has a zero minimum at $x$ and $p=D \varphi(x)$. Hence, $u$ is convex if and only if (3.3) holds in the viscosity sense. Notice that by taking $z=x$ the left side of (3.3) is identically zero.

Remark 3.2. One might suspect that the equation giving the convex envelope is

$$
\max \left\{u(x)+\max _{y \in \bar{\Omega}}[D u(x) \cdot(y-x)-u(y)], u(x)-g(x)\right\}=0, \quad x \in \Omega .
$$

However, (3.4) does not have a unique solution. For example if we take $g(x)=0,0 \leqslant x \leqslant \frac{1}{2}$, and $g(x)=1, \frac{1}{2} \leqslant x \leqslant 1$, it is obvious that

$$
g^{* *}(x)= \begin{cases}0, & \text { if } 0 \leqslant x \leqslant \frac{1}{2} \\ 1+2(x-1), & \text { if } \frac{1}{2} \leqslant x \leqslant 1\end{cases}
$$

and this solves (3.4). It is easy to check that

$$
u(x)= \begin{cases}0, & \text { if } 0 \leqslant x \leqslant \frac{3}{4} \\ 1+4(x-1), & \text { if } \frac{3}{4} \leqslant x \leqslant 1\end{cases}
$$

is also a solution. That means that (3.4) is not sufficient to uniquely characterize the convex envelope in the usual sense. Incidentally, one can make $g$ in this example continuous by connecting $(1 / 2,0)$ and $(1 / 2+\varepsilon, 1)$ with a steep linear piece.

On the other hand, $g^{* *}$ is the maximal subsolution of (3.4). To see that, if $v$ is any subsolution of (3.4), then $v \leqslant g$ on $\bar{\Omega}$ and

$$
v(x)+\max _{y \in \bar{\Omega}}[D v(x) \cdot(y-x)-v(y)] \leqslant 0,
$$

in the viscosity sense. Then Lemma 3.1 implies that $v$ is convex. Consequently, $v \leqslant g^{* *} \leqslant g$. Finally, $g^{* *}$ is clearly a subsolution of (3.4) again using Lemma 3.1.

The problem with (3.4) is that the equation seems to be coercive, but it is not coercive enough. We can fix that in the following way.

Let $\lambda>1$. Consider the Dirichlet problem with continuous boundary condition $u(x)=g(x)$ on $\partial \Omega$, and

$$
\lambda u(x)+\max _{y \in \bar{\Omega}}[D u(x) \cdot(y-x)-u(y)]=h(x), \quad x \in \Omega .
$$

The boundary condition is considered in the viscosity sense.

In what follows we shall use the notation

$$
F(x, u(x), p)=\max _{y \in \bar{\Omega}}[p \cdot(y-x)-(u(y)-u(x))] .
$$

Our problem becomes

$$
(\lambda-1) u(x)+F(x, u(x), D u(x))=h(x), \quad x \in \Omega, u(x)=g(x), x \in \partial \Omega .
$$


Remark 3.3. Since we do not want to carry along minus signs, a viscosity subsolution (supersolution) of (3.6) is an upper (lower) semicontinuous function $u$ such that $x \in \arg \max u-\varphi(x \in \arg \min u-\varphi)$ implies

$$
(\lambda-1) u(x)+F(x, u(x), D \varphi(x)) \leqslant h(x), \quad(\geqslant h(x)) .
$$

\section{These inequalities are reversed from the second order case in the first section.}

As for the boundary condition, if $x \in \arg \max u-\varphi$ with $x \in \partial \Omega$, then

$$
\min \{(\lambda-1) u(x)+F(x, u(x), D \varphi(x))-h(x), u-g\} \leqslant 0,
$$

and $x \in \arg \min u-\varphi, x \in \partial \Omega$,

$$
\max \{(\lambda-1) u(x)+F(x, u(x), D \varphi(x))-h(x), u-g\} \geqslant 0 .
$$

The next lemma gives a sufficient condition so that a given function coincides with it's convex envelope at the boundary. This lemma is due to Alvarez, Lasry and Lions [1].

Lemma 3.4. Let $\Omega \subset \mathbb{R}^{n}$ be a convex open bounded domain and $u: \bar{\Omega} \rightarrow \mathbb{R}$ lower semicontinuous. If either $\Omega$ is strictly convex or there is a convex, lower semicontinuous function $\Psi$ on $\bar{\Omega}$ such that $u=\Psi$ on $\partial \Omega$, then $u^{* *}=u$ on $\partial \Omega$.

We start by proving that (3.6) has only one viscosity solution. This follows from the following comparison theorem.

Theorem 3.5. Let $\Omega \subset \mathbb{R}^{n}$ be open and bounded. Suppose that $g, h: \bar{\Omega} \rightarrow \mathbb{R}$ is continuous; $u: \bar{\Omega} \rightarrow \mathbb{R}$ is an upper semicontinuous subsolution to (3.6); $v: \bar{\Omega} \rightarrow \mathbb{R}$ is a lower semicontinuous supersolution to (3.6); Then, $u(x) \leqslant v(x)$ for all $x \in \partial \Omega$ implies $u(x) \leqslant v(x)$ for all $x \in \Omega$.

Proof. For $\varepsilon>0$, consider the upper semicontinuous function $w_{\varepsilon}: \bar{\Omega} \times \bar{\Omega} \rightarrow \mathbb{R}$ given by

$$
w_{\varepsilon}(x, y)=u(x)-v(y)-\frac{1}{2 \varepsilon}|x-y|^{2}
$$

and pick $\left(x_{\varepsilon}, y_{\varepsilon}\right) \in \arg \max \left\{w_{\varepsilon}(x, y) \mid(x, y) \in \bar{\Omega} \times \bar{\Omega}\right\}$. When $\varepsilon \searrow 0, w_{\varepsilon}(x, y)$ converges pointwise to a function given by

$$
w_{\varepsilon}(x, y) \stackrel{\varepsilon \rightarrow 0}{\longrightarrow} \begin{cases}u(x)-v(x), & \text { if } x=y ; \\ -\infty, & \text { if } x \neq y .\end{cases}
$$

Consequently,

$$
\max _{(x, y) \in \bar{\Omega} \times \bar{\Omega}} w_{\varepsilon}(x, y) \rightarrow \max _{x \in \bar{\Omega}} u(x)-v(x)=u(\bar{x})-v(\bar{x}),
$$

with $\bar{x} \in \arg \max \{u(x)-v(x) \mid x \in \bar{\Omega}\}$. In particular for small enough $\varepsilon$,

$$
u\left(x_{\varepsilon}\right)-v\left(y_{\varepsilon}\right)-\frac{1}{2 \varepsilon}\left|x_{\varepsilon}-y_{\varepsilon}\right|^{2} \geqslant u(\bar{x})-v(\bar{x}) .
$$

Then, because $u$ is bounded above and $v$ is bounded below, the quantity $\frac{1}{2 \varepsilon}\left|x_{\varepsilon}-y_{\varepsilon}\right|^{2}$ is bounded above. In particular, $\left|x_{\varepsilon}-y_{\varepsilon}\right| \rightarrow 0$.

Consider a sequence $\varepsilon \searrow 0$ and, without loss of generality, suppose that sequences $\left\{x_{\varepsilon}\right\},\left\{y_{\varepsilon}\right\}$ are convergent, to some $x_{0}=y_{0}$. First, consider the case of $x_{0} \in \partial \Omega$. Then

$$
\begin{aligned}
\limsup u\left(x_{\varepsilon}\right)-v\left(y_{\varepsilon}\right)-\frac{1}{2 \varepsilon}\left|x_{\varepsilon}-y_{\varepsilon}\right|^{2} & \leqslant \limsup u\left(x_{\varepsilon}\right)-v\left(y_{\varepsilon}\right) \\
& \leqslant u\left(x_{0}\right)-v\left(y_{0}\right)=u\left(x_{0}\right)-v\left(x_{0}\right) \leqslant 0
\end{aligned}
$$

because we assumed $u(x) \leqslant v(x)$ for all $x \in \partial \Omega$. This and (3.7)-(3.8) implies that $\max _{x \in \bar{\Omega}} u(x)-v(x) \leqslant 0$, which says $u \leqslant v$ on $\bar{\Omega}$. 
Second, consider the case of $x_{0} \in \Omega$, which ensures that $x_{\varepsilon}, y_{\varepsilon} \in \Omega$ for small enough $\varepsilon$. Then $\varphi$ touches $u$ from above at $x_{\varepsilon}$ and $\psi$ touches $v$ from below at $y_{\varepsilon}$, where

$$
\varphi(x)=v\left(y_{\varepsilon}\right)+\frac{1}{2 \varepsilon}\left|x-y_{\varepsilon}\right|^{2}, \quad \psi(y)=u_{\sigma}\left(x_{\varepsilon}\right)-\frac{1}{2 \varepsilon}\left|x_{\varepsilon}-y\right|^{2} .
$$

Since $D \varphi\left(x_{\varepsilon}\right)=D \psi\left(y_{\varepsilon}\right)=\frac{1}{\varepsilon}\left(x_{\varepsilon}-y_{\varepsilon}\right)$, the assumptions about $u$ being a subsolution and $v$ being a supersolution imply

$$
(\lambda-1) u\left(x_{\varepsilon}\right)+F\left(x_{\varepsilon}, u\left(x_{\varepsilon}\right), \frac{1}{\varepsilon}\left(x_{\varepsilon}-y_{\varepsilon}\right)\right) \leqslant h\left(x_{\varepsilon}\right),
$$

and

$$
(\lambda-1) v\left(y_{\varepsilon}\right)+F\left(y_{\varepsilon}, v\left(y_{\varepsilon}\right), \frac{1}{\varepsilon}\left(x_{\varepsilon}-y_{\varepsilon}\right)\right) \geqslant h\left(y_{\varepsilon}\right) .
$$

Then, for all $z \in \bar{\Omega}$,

$$
u(z)-v(z) \leqslant u\left(x_{\varepsilon}\right)-v\left(y_{\varepsilon}\right)-\frac{1}{2 \varepsilon}\left|x_{\varepsilon}-y_{\varepsilon}\right|^{2} \leqslant u\left(x_{\varepsilon}\right)-v\left(y_{\varepsilon}\right),
$$

and consequently $u(z)-v(z) \leqslant u\left(x_{\varepsilon}\right)-v\left(y_{\varepsilon}\right)$ for all $z \in \bar{\Omega}$.

Then (3.9) implies

$$
\lambda u\left(x_{\varepsilon}\right)+\max _{z \in \bar{\Omega}}\left[\frac{1}{\varepsilon}\left(x_{\varepsilon}-y_{\varepsilon}\right) \cdot\left(z-x_{\varepsilon}\right)-u(z)\right]-h\left(x_{\varepsilon}\right) \leqslant 0,
$$

while (3.10) yields $z_{\varepsilon} \in \Omega$ such that

$$
\lambda v\left(y_{\varepsilon}\right)+\left[\frac{1}{\varepsilon}\left(x_{\varepsilon}-y_{\varepsilon}\right) \cdot\left(z_{\varepsilon}-y_{\varepsilon}\right)-v\left(z_{\varepsilon}\right)\right]-h\left(y_{\varepsilon}\right) \geqslant 0 .
$$

Use this $z_{\varepsilon}$ in (3.11)

$$
-\lambda u\left(x_{\varepsilon}\right)-\left[\frac{1}{\varepsilon}\left(x_{\varepsilon}-y_{\varepsilon}\right) \cdot\left(z_{\varepsilon}-x_{\varepsilon}\right)-u\left(z_{\varepsilon}\right)\right]+h\left(x_{\varepsilon}\right) \geqslant 0 .
$$

Add the two preceding inequalities results in

$$
\lambda\left[v\left(y_{\varepsilon}\right)-u\left(x_{\varepsilon}\right)\right]+\frac{1}{\varepsilon}\left|x_{\varepsilon}-y_{\varepsilon}\right|^{2}-\left[v\left(z_{\varepsilon}\right)-u\left(z_{\varepsilon}\right)\right] \geqslant h\left(y_{\varepsilon}\right)-h\left(x_{\varepsilon}\right) .
$$

Using $u\left(z_{\varepsilon}\right)-v\left(z_{\varepsilon}\right) \leqslant u\left(x_{\varepsilon}\right)-v\left(y_{\varepsilon}\right)$ we get

$$
\frac{1}{\varepsilon}\left|x_{\varepsilon}-y_{\varepsilon}\right|^{2} \geqslant h\left(y_{\varepsilon}\right)-h\left(x_{\varepsilon}\right)+(1-\lambda)\left(v\left(y_{\varepsilon}\right)-u\left(x_{\varepsilon}\right)\right) \text {. }
$$

We know that

$$
\max _{x \in \bar{\Omega}} u(x)-v(x)=u(\bar{x})-v(\bar{x}) \leqslant u\left(x_{\varepsilon}\right)-v\left(y_{\varepsilon}\right)-\frac{1}{2 \varepsilon}\left|x_{\varepsilon}-y_{\varepsilon}\right|^{2} \leqslant u\left(x_{\varepsilon}\right)-v\left(y_{\varepsilon}\right),
$$

which implies

$$
\frac{1}{2 \varepsilon}\left|x_{\varepsilon}-y_{\varepsilon}\right|^{2} \leqslant u\left(x_{\varepsilon}\right)-v\left(y_{\varepsilon}\right)-(u(\bar{x})-v(\bar{x})) .
$$

Since $x_{\varepsilon}-y_{\varepsilon} \rightarrow 0$, and $u\left(x_{\varepsilon}\right)-v\left(y_{\varepsilon}\right)-(u(\bar{x})-v(\bar{x})) \rightarrow 0$, we know that $\frac{1}{2 \varepsilon}\left|x_{\varepsilon}-y_{\varepsilon}\right|^{2} \rightarrow 0$. Sending $\varepsilon \rightarrow 0$ in (3.13) we get (using $\lambda>1$ )

$$
0 \geqslant(1-\lambda)(v(\bar{x})-u(\bar{x})) \quad \Longrightarrow \quad u(\bar{x})-v(\bar{x})=\max _{x \in \bar{\Omega}} u(x)-v(x) \leqslant 0,
$$

which says $u(x) \leqslant v(x), x \in \bar{\Omega}$. 
The proof of the following theorem is similar to the preceding.

Theorem 3.6. Let $u$ and $v$ denote upper (lower) semicontinuous subsolution (supersolution) of

$$
\begin{aligned}
& \frac{\partial u}{\partial t}+F(x, u(t, x), D u(t, x))=0, \quad(t, x) \in(0, \infty) \times \Omega, \\
& u(t, x)=g(x), \quad(t, x) \in(\{0\} \times \Omega) \cup([0, T] \times \partial \Omega), \forall T>0 .
\end{aligned}
$$

Then

$$
u \leqslant v, \quad(t, x) \in(\{0\} \times \Omega) \cup([0, T] \times \partial \Omega) \quad \Longrightarrow \quad u \leqslant v, \quad(t, x) \in[0, T] \times \Omega, \forall T>0 .
$$

\subsection{Construction of the convex envelope}

Let $\Omega \subset \mathbb{R}^{n}$ be a nonempty, bounded, convex, and open set. If $g: \bar{\Omega} \rightarrow \mathbb{R}$ is continuous, then $g^{* *}: \Omega \rightarrow \mathbb{R}$ the convex envelope of $g$, is also continuous. Furthermore, if we assume either that $\Omega$ is strictly convex or there is a lower semicontinuous convex $\Psi$ on $\bar{\Omega}$ so that $g=\Psi$ on $\partial \Omega$, then $g=g^{* *}$ on $\partial \Omega$.

Consider the problem

$$
\left.\begin{array}{ll}
u(x)+F(x, u(x), D u(x))=h(x), & x \in \Omega, \\
u(x)=g(x), & x \in \partial \Omega .
\end{array}\right\}
$$

This is (3.6) with $\lambda=2$.

Lemma 3.7. If $g \geqslant h \geqslant g^{* *}$ then (3.16) has a unique viscosity solution $u$ and $g^{* *} \leqslant u \leqslant g, x \in \bar{\Omega}$. If $g$ or $\Omega$ satisfies the conditions of Lemma 3.4, then $u=g=g^{* *}, x \in \partial \Omega$.

Proof. It is easy to check that the function $g$ is a supersolution since $g+F(x, g, D g) \geqslant g \geqslant h$. Furthermore, $g^{* *}$ is a subsolution since $g^{* *}+F\left(x, g^{* *}, D g^{* *}\right)=g^{* *} \leqslant h$. By Perron's method (which is easily extended to this problem, see [2], for example), there is a solution. Uniqueness follows from the comparison theorem as does the inequality $g^{* *} \leqslant u \leqslant g$. If $g$ or $\Omega$ satisfies the conditions of Lemma 3.4 then $u=g$ on $\partial \Omega$.

The next theorem provides an iterative method for construction of the convex envelope. It does have the drawback of requiring the solution of a sequence of nonlocal equations.

\section{Theorem 3.8. Let}

$$
u_{0}(x)=g(x), \quad x \in \bar{\Omega}
$$

and, for $n=0,1, \ldots$, let $u_{n+1}$ be the solution to

$$
\begin{aligned}
& u_{n+1}+F\left(x, u_{n+1}(x), D u_{n+1}(x)\right)=u_{n}, \quad x \in \Omega, \\
& u_{n+1}(x)=g(x), \quad x \in \partial \Omega .
\end{aligned}
$$

Then, for $n=1,2, \ldots, u_{n}$ is continuous, $g^{* *}(x) \leqslant u_{n}(x) \leqslant g(x)$ for all $x \in \bar{\Omega} ;$ the sequence $\left\{u_{n}\right\}$ is nonincreasing; and the function $W: \bar{\Omega} \rightarrow \mathbb{R}$ defined by

$$
W(x)=\lim _{n \rightarrow \infty} u_{n}(x)=\inf _{n=1,2, \ldots} u_{n}(x)
$$

satisfies $W(x)=g^{* *}(x)$ for every $x \in \Omega$. If $g$ or $\Omega$ satisfies the conditions of Lemma 3.4, then $u_{n}(x)=g^{* *}(x)=$ $g(x)=W(x), x \in \partial \Omega$.

Proof. It is standard that if $u_{n}$ solves the problem then $u_{n}$ is continuous on $\bar{\Omega}$ for $n=0,1, \ldots$ Indeed, if $u_{n}$ is a solution to (3.17) then the upper semicontinuous envelope $\left(u_{n}\right)^{*}(x) \leqslant g(x)$ and the lower semicontinuous envelope $\left(u_{n}\right)_{*}(x) \geqslant g(x)$ for all $x \in \partial \Omega$, and thus for such $x,\left(u_{n}\right)^{*}(x) \leqslant\left(u_{n}\right)_{*}(x)$. Then, if $u_{n-1}$ is continuous, Theorem 3.5 implies that $\left(u_{n}\right)^{*}(x) \leqslant\left(u_{n}\right)_{*}(x)$ for all $x \in \Omega$, and because $\left(u_{n}\right)_{*} \leqslant u_{n} \leqslant\left(u_{n}\right)^{*}$, it must be that $\left(u_{n}\right)^{*}=u_{n}=\left(u_{n}\right)_{*}$, which means that $u_{n}$ is continuous. 
Claim 1. The sequence $g^{* *} \leqslant u_{n} \leqslant g, n=0,1, \ldots$

By induction note that $g^{* *} \leqslant u_{0}=g$ and hence $u_{1}$ exists by Lemma 3.7 and $g^{* *} \leqslant u_{1} \leqslant g$. Now suppose $g^{* *} \leqslant$ $u_{n} \leqslant g$. Then by Lemma $3.7 u_{n+1}$ exists and $g^{* *} \leqslant u_{n+1} \leqslant g$.

Claim 2. The sequence $u_{n}$ is nonincreasing on $\Omega$.

Since $F(x, u(x), p) \geqslant 0$ for any $u$ and $p \in \mathbb{R}^{n}$, and $u_{n+1}$ is a subsolution to (3.17),

$$
u_{n+1}(x) \leqslant u_{n+1}(x)+F\left(x, u_{n+1}(x), D \varphi(x)\right) \leqslant u_{n}(x),
$$

for every smooth $\varphi$ touching $u_{n+1}$ from above. Thus $u_{n+1}(x) \leqslant u_{n}(x)$.

Claim 3. $W(x)=\lim _{n \rightarrow \infty} u_{n}(x)=\inf _{n=1,2, \ldots} u_{n}(x)$ is convex.

The limit defining $W$ exists and is finite, because the sequence $u_{n}$ is nonincreasing and bounded below by $g^{* *}$. The function $W$ is upper semicontinuous because $u_{n}$ are. To show that $W$ is convex suppose it is not. Then there exists a smooth $\varphi$ touching $W$ from above at $x \in \Omega$ so that

$$
F(x, W(x), D \varphi(x)) \geqslant \delta>0 .
$$

Then for some $z \in \bar{\Omega} D \varphi(x) \cdot(z-x)-(W(z)-W(x)) \geqslant \delta / 2$. Since $W=\lim _{n \rightarrow \infty} u_{n}$, there is a sequence $x_{n} \in \Omega$ such that $\varphi$ touches $u_{n}$ from above at $x_{n}, x_{n} \rightarrow x, \varphi\left(x_{n}\right) \rightarrow \varphi(x)$, and $u_{n}\left(x_{n}\right) \rightarrow W(x)$.

For large enough $n, D \varphi\left(x_{n}\right) \cdot\left(z-x_{n}\right)-\left(u_{n}(z)-u_{n}\left(x_{n}\right)\right)>\delta / 3$. Hence

$$
F\left(x_{n}, u_{n}\left(x_{n}\right), D \varphi\left(x_{n}\right)\right)>\frac{\delta}{3} .
$$

Because $u_{n}$ is a subsolution of (3.17),

$$
u_{n}\left(x_{n}\right)+F\left(x, u_{n}\left(x_{n}\right), D \varphi\left(x_{n}\right)\right) \leqslant u_{n-1}\left(x_{n}\right) \leqslant u_{n-1}\left(x_{n-1}\right)+\varphi\left(x_{n}\right)-\varphi\left(x_{n-1}\right),
$$

since $x_{n-1} \in \arg \max \left(u_{n-1}-\varphi\right)$ gives

$$
u_{n-1}\left(x_{n-1}\right)-\varphi\left(x_{n-1}\right) \geqslant u_{n-1}\left(x_{n}\right)-\varphi\left(x_{n}\right) \text {. }
$$

Thus

$$
\frac{\delta}{3}<F\left(x_{n}, u_{n}\left(x_{n}\right), D \varphi\left(x_{n}\right)\right) \leqslant u_{n-1}\left(x_{n-1}\right)-u_{n}\left(x_{n}\right)+\varphi\left(x_{n}\right)-\varphi\left(x_{n-1}\right) .
$$

Sending $n \rightarrow \infty$, the right-hand side of this inequality goes to 0 , because $u_{n}\left(x_{n}\right) \rightarrow W(x), \varphi$ is continuous, and $x_{n} \rightarrow x$. This is a contradiction and we conclude $W$ is convex.

From the preceding claims we know $g^{* *} \leqslant W \leqslant g$ and $W$ is convex. However, $g^{* *}$ is the greatest convex minorant of $g$ and hence $W=g^{* *}$.

In a similar way it is easy to establish the following.

Theorem 3.9. Let $u(t, x)$ denote the unique viscosity solution of

$$
\left.\begin{array}{l}
\frac{\partial u}{\partial t}+F(x, u(t, x), D u(t, x))=0, \quad(t, x) \in(0, \infty) \times \Omega, \\
u(0, x)=g(x), \quad x \in \bar{\Omega}, \quad u(t, x)=g(x), \quad(t, x) \in[0, \infty) \times \partial \Omega .
\end{array}\right\}
$$

Then $\lim _{t \rightarrow \infty} u(t, x)=g^{* *}(x)$.

Observe that the function $w(t, x)=g(x)$ is a supersolution, and $w(t, x)=g^{* *}(x)$ is a subsolution of (3.19) and an easy extension of Perron's method implies the existence of a viscosity solution to (3.19) with $g^{* *}(x) \leqslant u(t, x) \leqslant g(x)$, $x \in \bar{\Omega}, t \geqslant 0$. 
Remark 3.10. Vese in [13] has considered a second order construction of the convex envelope. The model considered is

$$
\begin{aligned}
& u_{t}=\sqrt{1+|D u|^{2}} \min \left\{0, \lambda_{1}\left(D^{2} u\right)\right\}, \quad(t, x) \in(0, \infty) \times \Omega, \\
& u(0, x)=g(x), \quad x \in\{0\} \times \Omega \cup[0, \infty) \times \partial \Omega .
\end{aligned}
$$

Vese proves that $\lim _{t \rightarrow \infty} u=g^{* *}$.

\section{References}

[1] O. Alvarez, J.M. Lasry, P.-L. Lions, Convex viscosity solutions and state constraints, 76 (1997) 265-288.

[2] M. Bardi, I. Capuzzo-Dolcetta, Optimal Control and Viscosity Solutions of Hamilton-Jacobi-Bellman Equations, with appendices by Maurizio Falcone and Pierpaolo Soravia, Systems Control Found. Appl., Birkhäuser Boston, Inc., Boston, MA, 1997.

[3] G. Barles, J. Busca, Existence and comparison results for fully nonlinear degenerate elliptic equations without zeroth-order term, Comm. Partial Differential Equations 26 (11-12) (2001) 2323-2337.

[4] E.N. Barron, R. Goebel, R. Jensen, The quasiconvex envelope through first order partial differential equations which characterize quasiconvexity of nonsmooth functions, Discrete Contin. Dyn. Syst. Ser. B 17 (2012) 1693-1706.

[5] E.N. Barron, R. Goebel, R. Jensen, Quasiconvex functions and viscosity solutions of partial differential equations, Trans. Amer. Math. Soc., http://dx.doi.org/10.1090/S0002-9947-2013-05760-1, in press.

[6] M.G. Crandall, H. Ishii, P.-L. Lions, User's guide to viscosity solutions of second order partial differential equations, Bull. Amer. Math. Soc. 27 (1992) 1-67.

[7] R. Kohn, S. Serfaty, A deterministic-control-based approach to motion by curvature, Comm. Pure Appl. Math. 59 (3) (2006) $344-407$.

[8] G. Lu, P. Wang, A uniqueness theorem for degenerate elliptic equations, in: Geometric Methods in PDE's, in: Lect. Notes Semin. Interdiscip. Mat., vol. 7, Semin. Interdiscip. Mat. (S.I.M.), Potenza, 2008, pp. 207-222.

[9] G. Lu, P. Wang, Infinity Laplace equation with non-trivial right-hand side, Electron. J. Differential Equations 77 (2010), 12 pp.

[10] G. Lu, P. Wang, A PDE perspective of the normalized infinity Laplacian, Comm. Partial Differential Equations 33 (10-12) (2008) 1788-1817.

[11] A. Oberman, L. Silvestre, The Dirichlet problem for the convex envelope, Trans. Amer. Math. Soc. 363 (11) (2011) 5871-5886.

[12] A. Oberman, The convex envelope is the solution of a nonlinear obstacle problem, Proc. Amer. Math. Soc. 135 (6) (2007) $1689-1694$.

[13] L. Vese, A method to convexify functions via curve evolution, Comm. Partial Differential Equations 24 (1999) $1573-1591$. 